\title{
On Solutions of the GRIMES Type in Spherical Polar Coordinates and Cylindrical Coordinates
}

\author{
by \\ H. Arakawa \\ Meteorological Research Institute \\ (Received 14 May, 1952)
}

\begin{abstract}
Solutions of the GRIMES type are derived in both spherical polar coordinates and cylindrical coordinates. It is pointed out that solutions of this type have the close relationship with the law of conservation of angular momentum.
\end{abstract}

\section{In the spherical polar coordinates}

GRIMES [1] showed that it was possible to obtain integrable equations of steady, frictionless motion allowing for the variation of the CoRIOLIS force with latitude. The solution obtained depended on the assumption of no variation of horizontal velocity with longitude $\lambda$, and was further limited by an approximation that $\sin \phi=\phi$ ( $\phi$ being the latitude) was made, and so was valid only within the tropics. CROSSLEY [2] has modified GRIMES's treatment to obtain more exact solution using spherical polar coordinates instead of Cartesian coordinates, but he neglected the metric terms in his derivation.

The exact equations for steady horizontal motion without friction may be written in spherical polar coordinates as follows:

$$
\left\{\begin{array}{l}
\frac{u}{a \cos \phi} \frac{\partial u}{\partial \lambda}+\frac{v}{a} \frac{\partial u}{\partial \phi}-\frac{\tan \phi}{a} u v-2 \Omega \sin \phi \cdot v=-\frac{1}{\rho a \cos \phi} \frac{\partial p}{\partial \lambda}, \\
\frac{u}{a \cos \phi} \frac{\partial v}{\partial \lambda}+\frac{v}{a} \frac{\partial v}{\partial \phi}+\frac{\tan \phi}{a} u^{2}+2 \Omega \sin \phi \cdot u=-\frac{1}{\rho a} \frac{\partial p}{\partial \phi},
\end{array}\right.
$$

together with the equation of continuity

$$
\partial(\rho u) / \partial \lambda+\partial(\rho v \cos \phi) / \partial \phi=0
$$

where all symbols having the same significance used by CROSSLEY are used throughout. Following GRIMES and CROSSLEY, we assume that there is no change of velocity with longitude,

$$
\partial u / \partial \lambda=\partial v / \partial \lambda=0
$$

so that the equations reduce with $\rho$ constant to 


$$
\left\{\begin{array}{c}
\frac{v}{a} \frac{\partial u}{\partial \phi}-\frac{\tan \phi}{a} u v-2 \Omega \sin \phi \cdot v=-\frac{1}{\rho a \cos \phi} \frac{\partial p}{\partial \lambda}, \\
\frac{v}{a} \frac{\partial v}{\partial \phi}+\frac{\tan \phi}{a} u^{2}+2 \Omega \sin \phi \cdot u=-\frac{1}{\rho a}-\frac{\partial p}{\partial \phi}, \\
\partial(v \cos \phi) / \partial \phi=0 .
\end{array}\right.
$$

The last of these equations then yields

$$
v=A / \cos \phi,
$$

where $A$ is an arbitrary constant, which can if required be interpreted as the northward component of velocity at the equator. The term $A / \cos \phi$ on the right of (6) means that the solution holds for low and middle latitudes. From equations (4) and (3) we have

(7) $\frac{\partial}{\partial \phi}\left(\frac{v \cdot \cos \phi}{a} \frac{\partial u}{\partial \phi}-\frac{\sin \phi}{a} u v-2 \Omega \sin \phi \cos \phi \cdot v\right)=-\frac{1}{\rho a} \frac{\partial^{2} p}{\partial \phi} \frac{1}{\partial \lambda}=-\frac{1}{\rho a} \frac{\partial}{\partial \lambda}\left(\frac{\partial p}{\partial \phi}\right)=0$, whence using (6) and integrating

$$
\frac{1}{a} \frac{\partial u}{\partial \phi}-\frac{\tan \phi}{a} u-2 \Omega \sin \phi=\frac{B}{a},
$$

$B$ being another arbitrary constant; a second integration then gives the solution

$$
\left\{\begin{array}{l}
u=B \tan \phi-\Omega a \cos \phi+C / \cos \phi, \\
v=A / \cos \phi,
\end{array}\right.
$$

where $A, B$ and $C$ are arbitrary constants, respectively. From (4) and (8) the pressure gradients are given by

$$
\left\{\begin{array}{l}
-\frac{1}{\rho a} \frac{\partial p}{\partial \lambda}=\frac{A B}{a}, \\
-\frac{1}{\rho a} \frac{\partial p}{\partial \phi}=\frac{\sin \phi}{a \cos ^{3} \phi}\left\{A^{2}+(B \sin \phi+C)^{2}-\Omega^{2} a^{2} \cos ^{4} \phi\right\},
\end{array}\right.
$$

whence

$$
-\frac{d p}{\rho}=A B d \lambda+\frac{\sin \phi}{\cos ^{3} \phi}\left\{A^{2}+(B \sin \phi+C)^{2}-\Omega^{2} a^{2} \cos ^{4} \phi\right\} d \phi
$$

Since the atmospheric pressure $p$ must be an one-valued continuous function of the longitude $\lambda$ and the latitude $\phi, B$ is necessarily equal to zero. The pressure distribution, now independent of longitude $\lambda$, is given by

$$
-\frac{p}{\rho}=\frac{A^{2}+C^{2}}{2 \cos ^{2} \phi}-\frac{\Omega^{2} a^{2}}{2}-\sin ^{2} \phi+\text { const. }
$$

Then GRIMES's solution in spherical polar form from (8) is

$$
\left\{\begin{array}{l}
u=C / \cos \phi-\Omega a \cos \phi, \\
v=A / \cos \phi
\end{array}\right.
$$

where $C$ is an arbitrary but usually positive constant, which can if required be in- 
terpreted as the eastward component of absolute velocity at the equator. The westerly component of velocity is minimum at the equator. It must be noted here that the first of the solutions expressed by (11) becomes

$$
\cos \phi(u+\Omega a \cos \phi)=\text { const, }
$$

which represents the law of conservation of the angular momentum. SAKURABA [3] obtained the GRIMES's solution of this type.

The equations of motion being of second degree, there are in general two independent solutions, but the condition (3) is restrictive. An alternative solution of (7) is given by $v=0$, whence from (4), $\partial p / \partial \lambda=0$, and the wind becomes gradient wind in the case of steady zonal motion, both wind and isobars being along the parallel of latitude, the pressure gradient $\partial \not \partial \phi \phi$ being arbitrary, i.e.,

$$
2 \Omega \sin \phi \cdot u+\frac{\tan \phi}{a} u^{2}=-\frac{1}{\rho a}-\frac{\partial p}{\partial \phi}, \quad v=0 .
$$

The solution corresponding to the pressure distribution (10) is

$$
u= \pm \sqrt{A^{2}+C^{2}} / \cos \phi-\Omega a \cos \phi, \quad v=0 .
$$

The upper sign gives $(u+\Omega a \cos \phi)=\sqrt{A^{2}+C^{2}} / \cos \phi$, and so yields an absolute velocity which is positive. The lower sign gives an absolute velocity which is negative, and so this is not usually available in the earth's atmosphere.

Of these two solutions (11) and (12), the gradient wind in the case of steady zonal motion (12) reduces to zero when the pressure gradient vanishes, but this is not true of the solution (11). The vector difference between (11) and (12) has components

$$
\left(C \mp \sqrt{A^{2}+C^{2}}\right) / \cos \phi, \quad A / \cos \phi .
$$

It is of interests to see that these dual solutions (11) and (12) give the same kinetic energy $\left(A^{2}+C^{2}\right) / 2 \cos ^{2} \phi$ if the absolute velocities $(u+\Omega a \cos \phi, v)$ are taken into consideration.

We might to say with CROSSLEY that if solutions of the GRIMES type are found to exist, it follows that the gradient of pressure by itself is not sufficient to determine the horizontal motion of air, at least in the tropics.

The stream lines for the GRIMES's solution of the type (11) are defined by $a \cos \phi d \lambda / u=a d \phi / v$, the integral of which is

$$
A \lambda+\Omega a \sin \phi-\frac{C}{2} \ln \frac{1+\sin \phi}{1-\sin \phi}=\text { const. }
$$

The stream lines of this type are shown graphically in Fig. 1 for selected values of $A$ and $C$. 


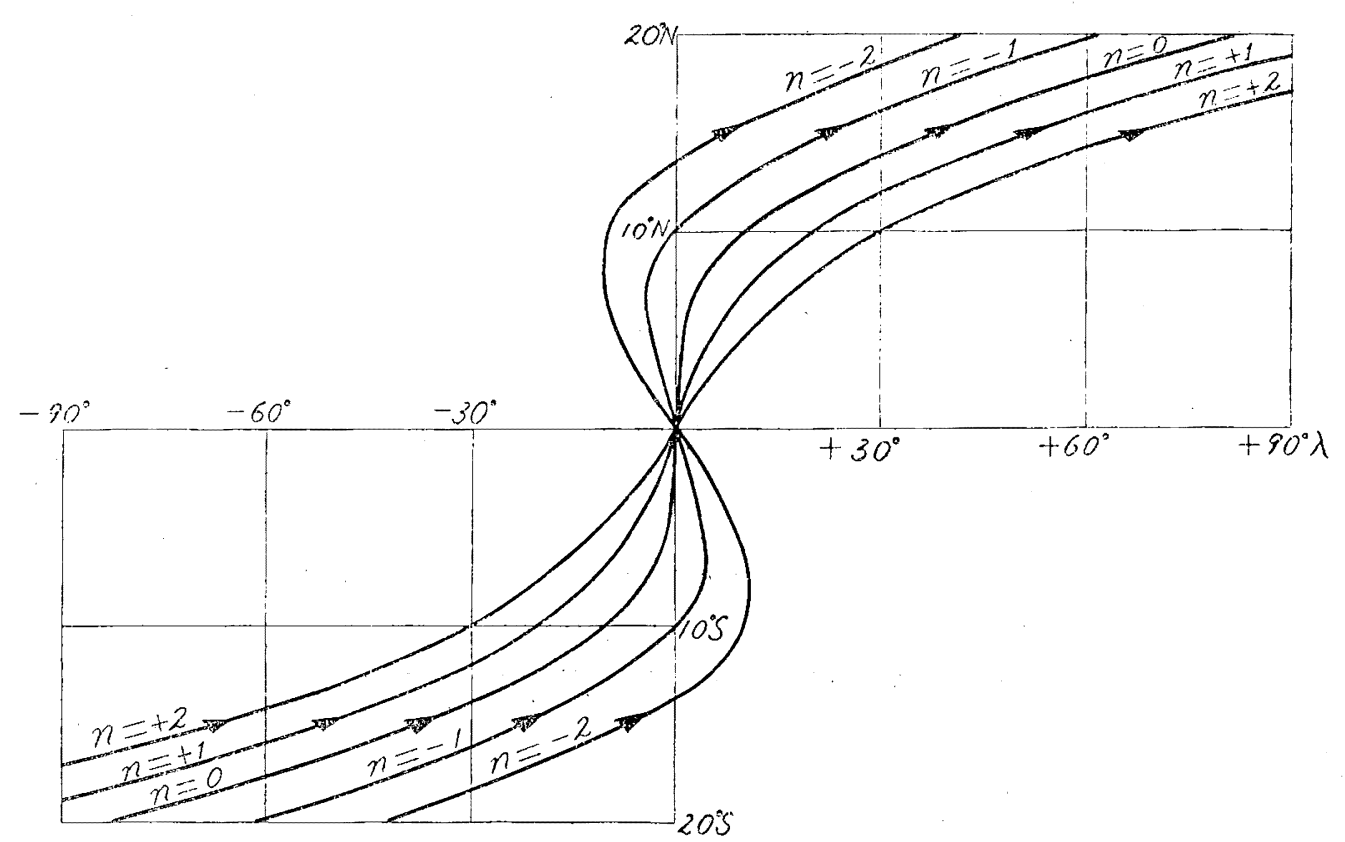

Fig. 1 (a).

$A=\Omega a / 100, \quad C=\Omega a+n A . \quad(n=-2,-1,0,+1,+2)$

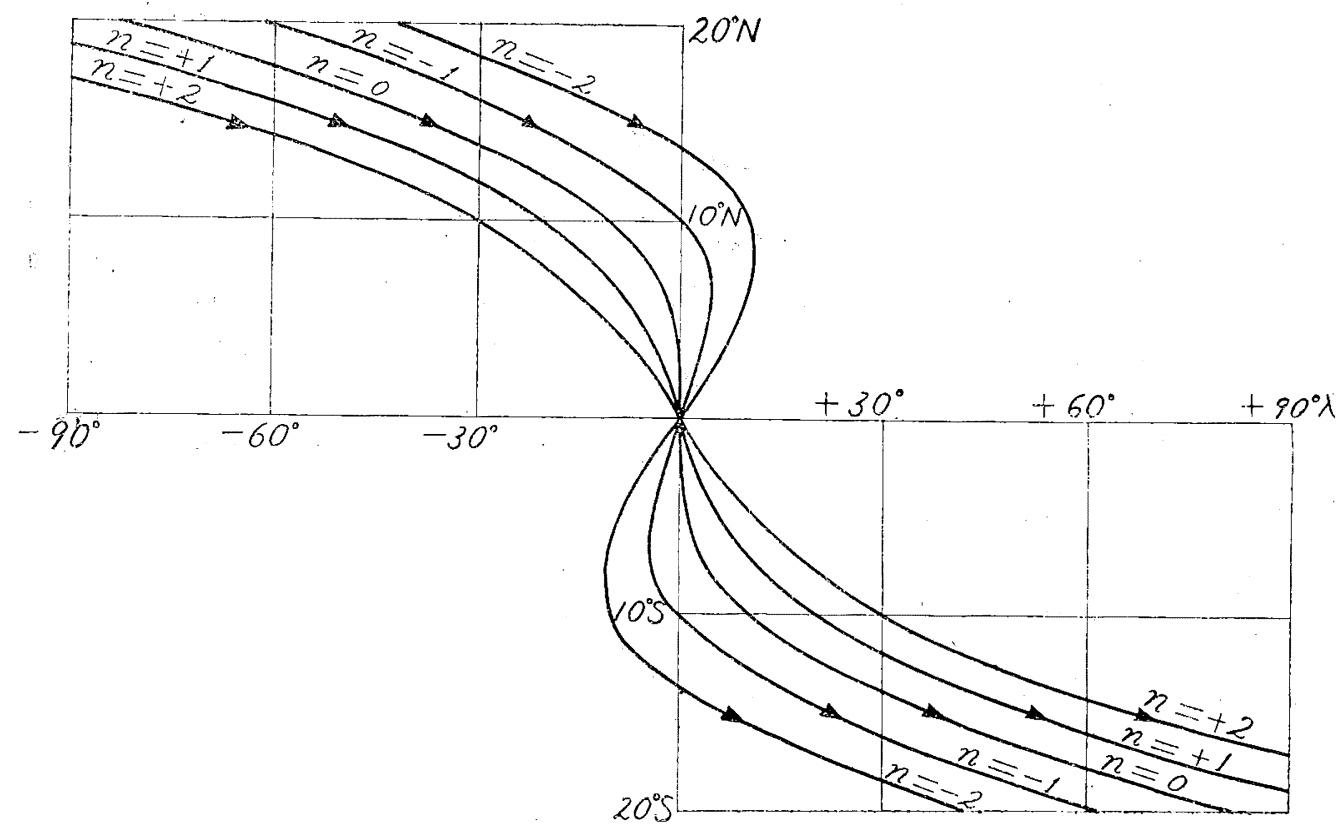

Fig. 1 (b).

$A=-\Omega a / 100, \quad C=\Omega a+n A(n=-2,-1,0,+1,+2)$ 


\section{In the cylindrical coordinates}

The dual solutions may be found for the steady horizontal motion of air in the system of concentric isobars, as tropical cyclones.

The exact equations for steady horizontal motion without friction may be written in cylindrical coordinates as follows:

$$
\left\{\begin{array}{l}
v_{r} \frac{\partial v_{\theta}}{\partial r}+\frac{v_{\theta}}{r} \frac{\partial v_{\theta}}{\partial \theta}+\frac{v_{\theta} v_{r}}{r}+2 \Omega \sin \phi \cdot v_{r}=-\frac{1}{\rho} \frac{\partial p}{r \partial \theta} \\
v_{r} \frac{\partial v_{r}}{\partial r}+\frac{v_{\theta}}{r} \frac{\partial v_{r}}{\partial \theta}-\frac{v^{\theta 2}}{r}-2 \Omega \sin \phi \cdot v_{\theta}=-\frac{1}{\rho} \frac{\partial p}{\partial r},
\end{array}\right.
$$

together with the equation of continuity

$$
\partial\left(\rho r v_{r}\right) / \partial r+\partial\left(\rho v_{\theta}\right) / \partial \theta=0 .
$$

Here $v_{r}, v_{\theta}$ are the radial and transverse components of the velocity at the point $(r, \theta)$, and other symbols have their same significance used in Section 1. Taking the origin at a proper position, we assume that the velocity is constant with azimuth

$$
\partial v_{r} / \partial \theta=\partial v_{\theta} / \partial \theta=0
$$

so that the equations reduce with $\rho=$ constant to

$$
\left\{\begin{array}{c}
v_{r} \frac{\partial v_{\theta}}{\partial r}+\frac{v_{\theta} v_{r}}{r}+2 \Omega \sin \phi \cdot v_{r}=-\frac{1}{\rho r} \frac{\partial p}{\partial \theta}, \\
v_{r} \frac{\partial v_{r}}{\partial r}-\frac{v_{\theta}^{2}}{r}-2 \Omega \sin \phi \cdot v_{\theta}=-\frac{1}{\rho} \frac{\partial p}{\partial r}, \\
\partial\left(r v_{r}\right) / \partial r=0 .
\end{array}\right.
$$

The last of these equations then yields

$$
v_{r}=\alpha \cdot r_{0} / r \text {, }
$$

where $\alpha$ is an arbitrary constant, which can if required be interpreted as the value of the radial component of velocity at the radius $r_{0}$. The term $\alpha \cdot r_{0} / r$ on the right of (19) means that the solution holds for the area except the origin. From equations (17) and (16) we have

$$
\frac{\partial}{\partial r}\left\{r v_{r}\left(\frac{\partial v_{\theta}}{\partial r}+\frac{v_{\theta}}{r}+2 \Omega \sin \phi\right)\right\}=-\frac{1}{\rho} \frac{\partial^{2} p}{\partial r \partial \theta}=-\frac{1}{\rho} \frac{\partial}{\partial \theta}\left(\frac{\partial p}{\partial r}\right)=0
$$

whence using (19) and integrating

$$
\partial v_{\theta} / \partial r+v_{\theta} / r+2 \Omega \sin \phi=\beta / r_{0},
$$

$\beta$ being another arbitrary constant; a second integration then gives the solution

$$
\left\{\begin{array}{l}
v_{\theta}=\frac{r_{0}}{r} \gamma+\left(\frac{\beta}{2 r_{0}}-\Omega \sin \phi\right) r, \\
v_{r}=\frac{r_{0}}{r} \alpha,
\end{array}\right.
$$

where $\alpha, \beta, \gamma$ are arbitrary constants. From (17) and (22) the pressure gradients are given by 


$$
\left\{\begin{array}{l}
-\frac{1}{\rho} \frac{\partial p}{r \partial \theta}=\frac{\alpha \beta}{r}, \\
-\frac{1}{\rho} \frac{\partial p}{\partial r}=-\frac{r_{0}^{2}}{r^{3}}\left(\alpha^{2}+\gamma^{2}\right)-\frac{\beta \gamma}{r}-\frac{\beta^{2} r}{4 r_{0}^{2}}+\Omega^{2} \sin ^{2} \phi \cdot r
\end{array}\right.
$$

whence

$$
-\frac{d p}{\rho}=\alpha \beta d \theta+\left\{\Omega^{2} \sin ^{2} \phi \cdot r-\frac{r_{0}{ }^{2}}{r^{3}}\left(\alpha^{2}+\gamma^{2}\right)-\frac{\beta \gamma}{r}-\frac{\beta^{2} r}{4 r_{0}^{2}}\right\} d r .
$$

Since the pressure $p$ must be an one-valued continuous function, $\beta$ is necessarily zero. The pressure distribution, now independent of azimuth, is given by

$$
-\frac{p}{\rho}=\mathrm{const}+\frac{\Omega^{2} \sin ^{2} \phi}{2} r^{2}+\frac{r_{0}^{2}}{2 r^{2}}\left(\alpha^{2}+\gamma^{2}\right) .
$$

Then GRIMES's solution from (22) is

$$
\left\{\begin{array}{l}
v_{\theta}=\gamma r_{0} / r \cdots \Omega \sin \phi \cdot r, \\
v_{r}=\alpha r_{0} / r
\end{array}\right.
$$

where $\gamma$ is an arbitrary but usually positive constant, which can if required be interpreted as the tangential component of absolute velocity at $r=r_{0}$. It might be noted here that the first of the solutions expressed by (24) becomes

$$
r\left(v_{0}+\Omega \sin \phi \cdot r\right)=\text { const, }
$$

which represents the law of conservation of the angular momentum.

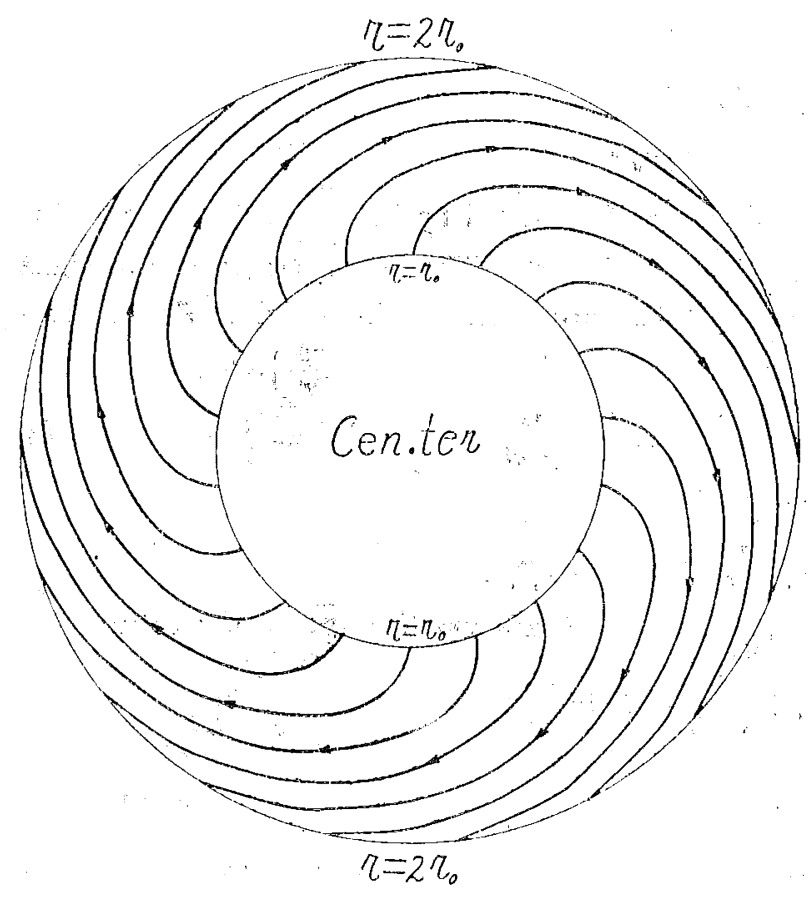

Fig. 2 (a). $\alpha=\Omega r_{0} \sin \phi / 2, \quad \gamma=\Omega \Omega r_{0} \sin \phi$

The equations of motion being of second degree, there are in general two independent solutions, but the condition (16) is restrictive. An alternative solution of (20) is given by $v_{r}=0$, whence from (17), $\partial p / \partial \theta=0$, and the wind becomes gradient wind in the case of steady circular motion, both wind and isobars being along the concentric circle, the pressure gradient $\partial p / \partial r$ being arbitrary, i. e.,

$2 \Omega \sin \phi \cdot v_{\theta}+\frac{v_{\theta}^{2}}{r}-\frac{1}{\rho} \frac{\partial p}{\partial r}=0$.

The solution corresponding to the pressure distribution (23) is

(25) $v_{\theta}= \pm r_{0} \sqrt{\alpha^{2}+\gamma^{2}} / r-\Omega r \sin \phi$, $v_{r}=0$.

The upper sign gives 


$$
\left(v_{\theta}+\Omega r \sin \phi\right)=r_{0} \sqrt{\alpha^{2}+\gamma^{2}} / r,
$$

and so yields an absolute velocity which is positive. The lower sign gives an absolute velocity which is negative, and so this is not usually available in the earth's atmosphere.

Of these two solutions (24) and (25), the gradient wind reduces to zero when the pressure gradient vanishes, but this is not true of the solution (24). The vector difference between (24) and (25) has the components

$$
\left(\gamma \mp \sqrt{\left.\alpha^{2}+\gamma^{2}\right)} r_{0} / r, \alpha r_{0} / r .\right.
$$

It is of interests to see that the dual solutions (24) and (25) give the same kinetic energy

$$
r_{0}^{2}\left(\alpha^{2}+\gamma^{2}\right) / 2 r^{2},
$$

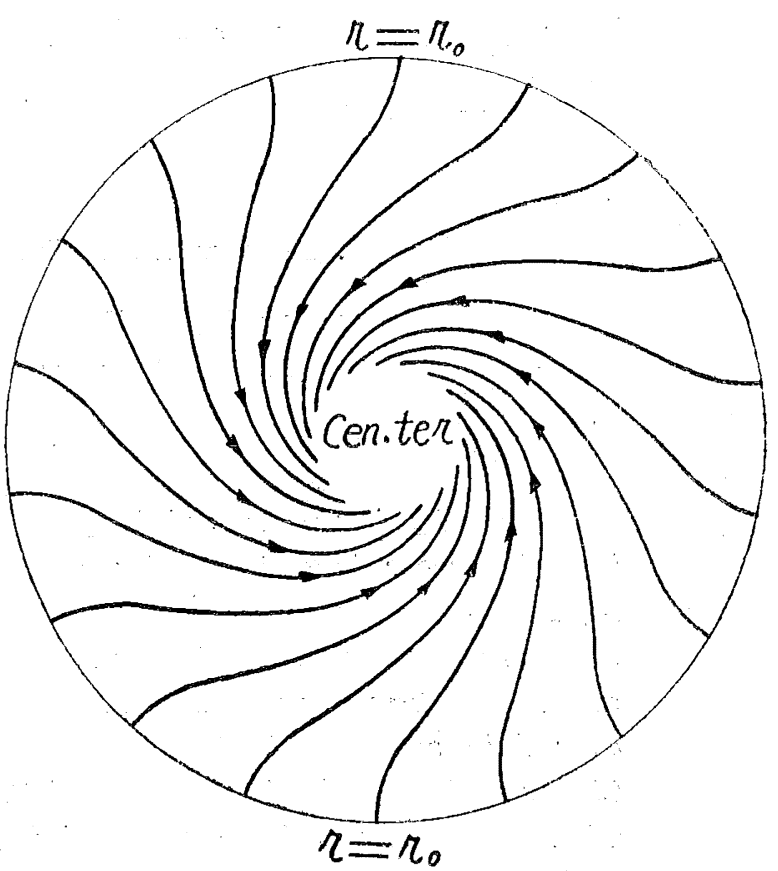

Fig. 2 (b). $\alpha=-\Omega r_{0} \sin \phi_{i} 2, \gamma=\Omega r_{0} \sin \phi$.

if the absolute velocities $\left(v_{\theta}+\Omega r \sin \phi, v_{r}\right)$, are taken into consideration.

The stream lines for the GRIMES's solution of the type (24) are defined by $d r / v_{r}=r d \theta / v_{\theta}$, the integral of which is

$$
\theta=(\gamma / \alpha) \log r-\left(\Omega r^{2} / 2 \alpha r_{0}\right) \sin \phi+\text { const. }
$$

The stream lines of this type are shown graphically in Fig. 2 for selected values of $\alpha$ and $\gamma$.

\section{References}

[1] Grimes, A., 1938: Mem. Malayan Meteorological Service, Singapore, No. 2.

Grimes, A., 1951: Compendium of Meteorology, p.881, published by the American Meteorological Society, Boston, Mass..

[2] Crossley, A. F., 1948: Quarterly Journal of the Royal Meteorological Society, London, 74, p. 379.

[3] Sakuraba, S., 1942: Journal of the Meteorological Society of Japan, 2nd Series, 20, p. 383. 